\title{
Phase-Locked Loop Stability Based on Stochastic Bounds
}

\author{
Robert J. A. Baker • Bosco Leung • \\ Christopher Nielsen
}

Received: date / Accepted: date

\begin{abstract}
In this paper we study the stability of a phase-locked loop (PLL) in the presence of noise. We represent the noise as Brownian motion and model the circuit as a nonlinear stochastic differential equation, with the noise lumped at the phase detector input. We show that for the PLL, the theory of asymptotics of singular diffusions can be applied and we use this theory to develop a new figure of merit which we call a stability margin. The stability margin provides easily computable bounds on the acceptable noise levels for which stability is guaranteed. Through simulation, we show that such a sufficient bound provides a realistic prediction for PLL stability.
\end{abstract}

Keywords phase-locked loops $\cdot$ stability $\cdot$ noise $\cdot$ stochastic differential equations

\section{Introduction}

A phase-locked loop (PLL) is a circuit that synchronizes its output signal with the phase and frequency of its reference signal. Phase-locked loops are used in clock generation, frequency synthesis and modulation [1,2,3]. Generally, a PLL is composed of a phase detector, loop filter, voltage-controlled oscillator (VCO) and a feedback loop containing a divide-by- $N$ counter for frequency synthesis (Figure 1). In the ideal synchronized or locked state, the phase error between the reference and output signals is practically zero and the frequency error is zero. However, in the presence of noise, this tracking is degraded and the signals may not lock exactly. In practice, designers account for the presence of noise by placing the system poles further into the left-half plane and by making conservative design decisions [4,5].

Due to the nonlinearity of the PLL while achieving phase lock, its stochastic stability is still an active area of research $[6,7,8]$. In $[6]$ the authors use reachability

Department of Electrical and Computer Engineering University of Waterloo

200 University Ave. West, Waterloo, Ontario, N2L 3G1, Canada.

Tel.: +1.519.888.4567 x 32678, Fax: +1.519.888.3077

\{rjabaker; bleung; cnielsen\}@uwaterloo.ca 
analysis to adjust the PLL lock range and adjust initial inputs, such as phase error. Mehrotra [7] derives a general expression for the PLL output spectrum using a stochastic differential equation (SDE). Both [6] and [7] are useful as simulation tools, but they do not explicitly investigate the PLL's stability. Another application of SDE models in [8] uses estimation to derive the PLL stability conditions, although the derivation uses formal stochastic methods and is not easily accessible to typical circuit designers. In this paper, we focus on PLL stability in the presence of noise and, following $[6,7,8]$, we focus on thermal noise. We represent the noise as Brownian motion and model the circuit as a nonlinear stochastic differential equation, with the noise lumped at the phase detector input. We then apply the asymptotics of singular diffusions discussed in [9] to develop a new figure of merit called the stability margin. The stability margin provides easily computable bounds on the acceptable noise levels for which stability is guaranteed. Through simulation, we show that such a sufficient bound provides a realistic prediction for PLL stability.

This paper is organized as follows. Section 2 first models the PLL as a nonlinear time-invariant system. Noise is then introduced to the model following [9] and the PLL is described as a diffusion process using an SDE. This model allows the introduction of Theorem 1 ([9, Theorem 4.1]), which provides sufficient conditions for the PLL to be stable in the presences of input-referred noise. In Section 3, the PLL system is linearized and we show that for a multiplier type phase detector and a passive lead-lag filter the hypotheses of Theorem 1 hold. A new figure of merit called the stability margin is introduced. It depends on noise power levels and the linearized system dynamics and is used to guarantee the stochastic stability of the PLL. In Section 4, the use of the stability margin is first illustrated on an RLC circuit. It is then illustrated on four PLL configurations. The theoretical predictions are compared to a simple circuit, and the time domain simulations are verified. Section 5 presents conclusions.

\section{System Model and Stability Bounds}

A block diagram of a PLL is shown in Figure 1. We assume a multiplier type phase

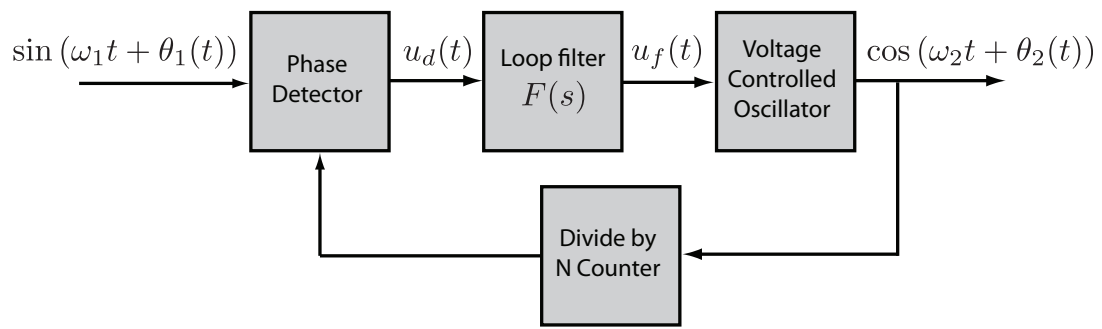

Fig. 1: Block diagram of a phase-locked loop.

detector. Using the familiar identity

$$
\begin{aligned}
2 \sin \left(\omega_{1} t+\theta_{1}(t)\right) \cos \left(\omega_{2} t+\theta_{2}(t)\right) & =\sin \left(\left(\omega_{1}+\omega_{2}\right) t+\theta_{1}(t)+\theta_{2}(t)\right) \\
& +\sin \left(\left(\omega_{1}-\omega_{2}\right) t+\theta_{1}(t)-\theta_{2}(t)\right),
\end{aligned}
$$


we make two fundamental assumptions [10] that lead to a common model of a PLL.

(1) The first term on the right side of the equality (1) is attenuated by the lowpass filter.

(2) The reference and output frequencies are approximately equal, $\omega_{1} \approx \omega_{2}$, so that difference can be incorporated into $\theta_{2}(t)$.

These assumptions mean that the VCO can be modelled as an integrator [1] and leads to the model in Figure 2. We now proceed to find a nonlinear state model for

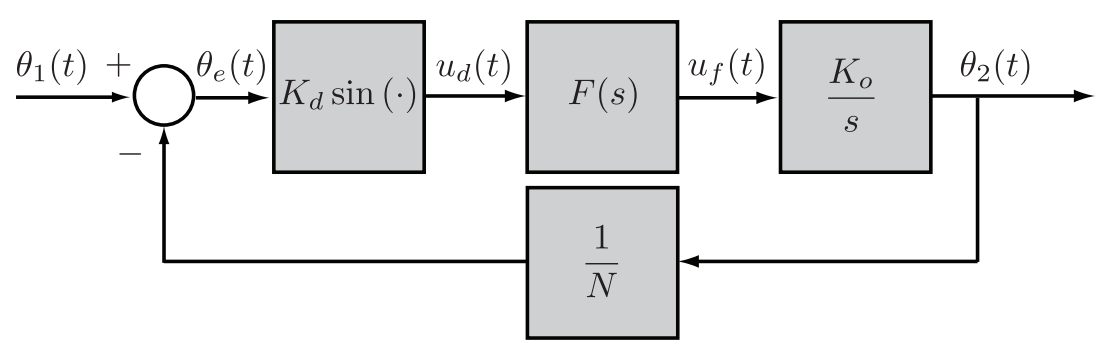

Fig. 2: Simplified block diagram of a phase-locked loop.

the system in Figure 2. We denote the strictly proper state model of the VCO by the 4-tuple of matrices $\left(A_{o}, B_{o}, C_{o}, D_{o}\right)$ where, for the integrator, $A_{o}=0, B_{o}=1$, $C_{o}=K_{o}, D_{o}=0$. We denote the minimal state model of the low pass filter by the 4-tuple of matrices $\left(A_{f}, B_{f}, C_{f}, D_{f}\right)$. The dimension of the square matrix $A_{f}$ equals the order of the filter $F(s)$. Then the state model of the cascade connection between the filter and a strictly proper VCO is given by

$$
\begin{aligned}
\dot{x}(t) & =\left[\begin{array}{cc}
A_{f} & 0 \\
B_{o} C_{f} & A_{o}
\end{array}\right] x(t)+\left[\begin{array}{c}
B_{f} \\
B_{o} D_{f}
\end{array}\right] u_{d}(t) \\
\theta_{2}(t) & =\left[\begin{array}{ll}
0 & C_{o}
\end{array}\right] x(t)
\end{aligned}
$$

where the state $x(t)=\left(x_{1}(t), x_{2}(t)\right)$ is comprised of the state of the filter $\left(x_{1}(t)\right)$ and the state of the $\operatorname{VCO}\left(x_{2}(t)\right)$.

Example 1 For the case of a passive lead-lag filter

$$
\frac{U_{d}(s)}{U_{f}(s)}=F(s)=\frac{1+\tau_{2} s}{1+s\left(\tau_{1}+\tau_{2}\right)}=\frac{1-\frac{\tau_{2}}{\tau_{1}+\tau_{2}}}{1+s\left(\tau_{1}+\tau_{2}\right)}+\frac{\tau_{2}}{\tau_{1}+\tau_{2}}
$$

we get

$$
A_{f}=-\frac{1}{\tau_{1}+\tau_{2}}, B_{f}=1, C_{f}=\frac{\tau_{1}}{\left(\tau_{1}+\tau_{2}\right)^{2}}, D_{f}=\frac{\tau_{2}}{\tau_{1}+\tau_{2}} .
$$

When connected in cascade with the integrator model of the VCO equation (2) becomes

$$
\begin{aligned}
& \dot{x}(t)=\left[\begin{array}{cc}
-\frac{1}{\tau_{1}+\tau_{2}} & 0 \\
\frac{\tau_{1}}{\left(\tau_{1}+\tau_{2}\right)^{2}} & 0
\end{array}\right] x(t)+\left[\begin{array}{c}
1 \\
\frac{\tau_{2}}{\tau_{1}+\tau_{2}}
\end{array}\right] u_{d}(t) \\
& \theta_{2}(t)=\left[\begin{array}{ll}
0 & K_{o}
\end{array}\right] x(t)
\end{aligned}
$$

where $x(t)=\left(x_{1}(t), x_{2}(t)\right) \in \mathbb{R}^{2}$. 
From (2) we immediately obtain that the closed-loop system is modelled by

$$
\begin{aligned}
\dot{x}(t) & =\left[\begin{array}{cc}
A_{f} & 0 \\
B_{o} C_{f} & A_{o}
\end{array}\right] x(t)+\left[\begin{array}{c}
B_{f} \\
B_{o} D_{f}
\end{array}\right] K_{d} \sin \left(\theta_{1}(t)-\frac{1}{N} \theta_{2}(t)\right) \\
\theta_{2}(t) & =\left[\begin{array}{ll}
0 & C_{o}
\end{array}\right] x(t) .
\end{aligned}
$$

If we treat the phase error $\theta_{e}:=\theta_{1}-\theta_{2}$ as the system output then the above system can be written

$$
\begin{aligned}
\dot{x}(t) & =\left[\begin{array}{cc}
A_{f} & 0 \\
B_{o} C_{f} & A_{o}
\end{array}\right] x(t)+\left[\begin{array}{c}
B_{f} \\
B_{o} D_{f}
\end{array}\right] K_{d} \sin \left(\theta_{1}(t)-\frac{1}{N}\left[0 C_{o}\right] x(t)\right) \\
\theta_{e}(t) & =-\frac{1}{N}\left[0 C_{o}\right] x(t)+\theta_{1}(t) .
\end{aligned}
$$

System (4) is a nonlinear time-invariant system with input $\theta_{1}$ and output $\theta_{e}$ of the form

$$
\begin{aligned}
\dot{x}(t) & =A x(t)+B \sin \left(\theta_{1}(t)+C x(t)\right) \\
\theta_{e}(t) & =C x(t)+\theta_{1}(t)
\end{aligned}
$$

where the dimensions of the constant matrices $A, B, C$ depend on the order of the lowpass filter.

Example 2 Re-visiting Example 1, in the case of the passive lead-lag filter the overall system (5) is given by

$$
\begin{aligned}
\dot{x}(t) & =\left[\begin{array}{cc}
-\frac{1}{\tau_{1}+\tau_{2}} & 0 \\
\frac{\tau_{1}}{\left(\tau_{1}+\tau_{2}\right)^{2}} & 0
\end{array}\right] x(t)+\left[\begin{array}{c}
1 \\
\frac{\tau_{2}}{\tau_{1}+\tau_{2}}
\end{array}\right] K_{d} \sin \left(\theta_{1}(t)-\left[0 K_{o} / N\right] x(t)\right) \\
\theta_{e}(t) & =-\left[0 K_{o} / N\right] x(t)+\theta_{1}(t) .
\end{aligned}
$$

\subsection{Noise Model}

The model (5) assumes no noise sources in the circuit. One of the most important properties of a PLL is its ability to suppress noise. Typically, the VCO and divideby- $\mathrm{N}$ counter both contribute a significant portion of noise to the PLL, including both thermal/white and $1 / f$ noise [11]. In the model developed in this section, we follow [7] and consider white noise. Following [9] we treat the noisy system as a diffusion process modelled by an SDE of the form

$$
\begin{aligned}
\mathrm{d} X(t) & =f\left(X(t), \theta_{1}(t)\right) \mathrm{d} t+\sigma(X(t)) \mathrm{d} W(t) \\
\theta_{e}(t) & =C X(t)+\theta_{1}(t) .
\end{aligned}
$$

Here $X(t)$ is the $k$-dimensional state vector in (5), $f\left(X(t), \theta_{1}(t)\right):=A X(t)+$ $B \sin \left(\theta_{1}(t)+\frac{1}{N} C X(t)\right), W(t)$ is a standard $k$-dimensional Brownian motion and $\sigma(X(t)) \in \mathbb{R}^{k \times k}$. The term $\sigma(\cdot)$ models the manner in which noise enters the circuit and is taken to be

$$
\sigma(X)=\left[\begin{array}{cc}
0 & 0 \\
0 & \sigma_{o}(X)
\end{array}\right] .
$$


The function $\sigma_{o}$ models the way noise enters the VCO. When the VCO has a first order model, $\sigma_{o}$ is a real-valued function.

Given a PLL model (6), we are interested in finding a bound on $\sigma(\cdot)$ under which stability of the circuit can be guaranteed. The bound is a function of both the noise power levels and the poles of the linearized system. Stability in this context means that the expectation of the solution to (6) is bounded and independent of the initial conditions. The next section elaborates and describes the main tool used to achieve such a characterization.

\subsection{Stability Bounds and Asymptotics of Singular Diffusions}

The main reference for this section is [9, Chapter 4]. Consider an SDE of the form

$$
\mathrm{d} X(t)=f(X(t)) \mathrm{d} t+\sigma(X(t)) \mathrm{d} W(t)
$$

where $X(t) \in \mathbb{R}^{k}, f$ is continuously differentiable and $\{W(t)\}$ is a $k$-dimensional standard Brownian motion. An example of a singular diffusion ${ }^{1}$ SDE is the OrnsteinUhlenbeck process. In terms of circuits, the Ornstein-Uhlenbeck process can be viewed as a model of an $\mathrm{RC}$ network with noise. The result in this section investigates the asymptotics of noise behaviour, i.e., that the noise power spectral density is bounded.

Let

$$
J(X):=\frac{\partial f}{\partial X}
$$

denote the Jacobian of the vector field $f$. The next result motivates the analysis of this paper.

Theorem 1 ([9, Theorem 4.1]) Let $\sigma(\cdot)$ be globally Lipschitz ${ }^{2}$ on $\mathbb{R}^{k}$, i.e.,

$$
\left(\exists \lambda_{0} \geq 0\right)\left(\forall x, y \in \mathbb{R}^{k}\right)\|\sigma(x)-\sigma(y)\| \leq \lambda_{0}\|x-y\| .
$$

Assume that, for all $x$, the eigenvalues of $J_{\mathrm{sym}}(x):=\frac{1}{2}\left(J(x)+J^{\top}(x)\right)$ are less than or equal to $-\lambda_{1}<0$, where $k \lambda_{0}^{2}<2 \lambda_{1}$. Then there exists a unique invariant distribution $\pi(\mathrm{d} z)$ for the diffusion, and $p(t ; x, \mathrm{~d} z)$ converges weakly to $\pi(\mathrm{d} y)$ as $t \rightarrow \infty$, for every $x \in \mathbb{R}^{k}$.

The proof of Thoerem 1 is based on the Ito integral $[12,13]$. If the conditions of Theorem 1 hold, then for any initial condition $X(0)=X_{0} \in \mathbb{R}^{k}$ of (8), the associated solution $X\left(t, X_{0}\right)$ satisfies

$$
\sup _{t \geq 0} \mathbb{E}\left[\left\|X\left(t, X_{0}\right)\right\|\right]<\infty
$$

where $\mathbb{E}[\cdot]$ is the expectation operator and sup is the supremum or least upper bound, equivalent to the maximum in the context of this paper. Furthermore, for any other initial condition $X_{0}^{\prime}$

$$
\lim _{t \rightarrow \infty} \mathbb{E}\left[\left\|X\left(t, X_{0}\right)-X\left(t, X_{0}^{\prime}\right)\right\|\right]=0 .
$$

\footnotetext{
1 Singular diffusion simply means that the matrix valued function $\sigma: \mathbb{R}^{k} \rightarrow \mathbb{R}^{k \times k}$ in (8) is singular, i.e., for all $X \in \mathbb{R}^{k}$, $\operatorname{det}(\sigma(X))=0$.

$2\|\cdot\|$ can be any vector norm but is taken to be the Euclidean norm in this paper.
} 
In the context of the PLL (6), Theorem 1 can be used to predict stochastic stability. If, under suitable assumption, the hypotheses of Theorem 1 hold for (6), then we conclude that the expectation of its solutions are bounded and that the solution does not depend on how the system is initialized. With the hypotheses holding, Theorem 1 says that the probability distribution converges as $t \rightarrow \infty$. In essence, the existence of a converged distribution is indicative of the stochastic stability of the system $[9,12,13]$. Furthermore, by taking the logical negation of the theorem statement, the analysis provides a necessary condition for the onset of instability.

We first compute the Jacobian (9) of the vector field in (6) and compute the eigenvalues of $J_{\text {sym }}=\frac{1}{2}\left(J+J^{\top}\right)$. The matrix $J_{\text {sym }}$, being real and symmetric, has only real eigenvalues. Next, assuming that they are all negative, we write them in order of magnitude

$$
-\lambda_{n} \leq \cdots \leq-\lambda_{1}<0
$$

From $\lambda_{1}$ above we compute largest allowable Lipschitz constant $\lambda_{0}$ using

$$
k \lambda_{0}^{2}<2 \lambda_{1}
$$

where the number $k$ depends on the order of the low pass filter used. Then, making physically motivated assumptions on the function $\sigma(\cdot)$, we use $\lambda_{0}$ to approximate an upper bound on the allowable noise levels that ensure stability. This program is undertaken in the next section for a PLL.

\section{Stability Analysis}

We begin by computing the Jacobian (9) of the vector field (5). We linearize the system at the locked state, i.e., $\theta_{e}=0$, because in that case the Jacobian does not depend on the state of (5) and we do not need to compute the associated equilibrium state. The subsequent linear model is a good approximation of (5) if $\left|\theta_{e}\right|$ is sufficiently small. For $f\left(x(t), \theta_{1}(t)\right):=A x(t)+B \sin \left(\theta_{1}(t)+C x(t)\right)$ we obtain

$$
J(x)=\frac{\partial f}{\partial x}=A+K_{d} \cos \left(\theta_{e}\right) B C=\left[\begin{array}{cc}
A_{f} & 0 \\
B_{o} C_{f} & A_{o}
\end{array}\right]-\frac{K_{d}}{N}\left[\begin{array}{c}
B_{f} \\
B_{o} D_{f}
\end{array}\right]\left[\begin{array}{ll}
0 & C_{o}
\end{array}\right] .
$$

For the sake of completeness we compute the linearization of (4) at $\theta_{e}=0$

$$
\begin{aligned}
\dot{\delta x}(t) & =J(x) \delta x(t)+\frac{K_{d}}{N}\left[\begin{array}{c}
B_{f} \\
B_{o} D_{f}
\end{array}\right] \delta \theta_{1}(t) \\
\delta \theta_{e}(t) & =-\frac{1}{N}\left[0 C_{o}\right] \delta x(t)+\delta \theta_{1}(t) .
\end{aligned}
$$

The associated input-output model is given by

$$
\frac{\Theta_{e}(s)}{\Theta_{1}(s)}=H(s)=-\frac{K_{d}}{N^{2}}\left[0 C_{o}\right](s I-J(x))^{-1}\left[\begin{array}{c}
B_{f} \\
B_{o} D_{f}
\end{array}\right] .
$$

In order to apply Theorem 1 to system (6) we compute, using (11), the matrix $J_{\text {sym }}(x)=\frac{1}{2}\left(J(x)+J^{\top}(x)\right)$. Let $-\lambda_{1}<0$ denote its largest eigenvalue. Using (10) we compute $\lambda_{0}$. Using this number we define a quantity, the stability margin, as

$$
\mathrm{SM}:=\lambda_{0}\|x-y\|-\|\sigma(x)-\sigma(y)\|
$$


where $x$ and $y$ are different points in the state space of (6) and $\sigma$ is given by (7). We predict the PLL to be stable if $\mathrm{SM}>0$.

\subsection{Coordinate Changes}

One issue in the above approach is that it may happen that $J_{\text {sym }}$ does not have all negative eigenvalues, even when $J(x)$ is Hurwitz. If the original system is governed by a linear SDE then [9, Proposition 4.2] can be used to reach the same conclusion as Theorem 1. Unfortunately that result does not provide the useful metric (14) for measuring stability margin. We overcome this problem by applying a linear coordinate change to the linearized model (12). The model (12) is generically controllable [14], that is, for almost any choice of the parameters, the matrix pair of $J(x)$ and

$$
\frac{K_{d}}{N}\left[\begin{array}{c}
B_{f} \\
B_{o} D_{f}
\end{array}\right]
$$

are controllable. The following result is standard. It states that there exists a linear change of coordinates that takes a controllable linear system into controllable canonical form.

Theorem 2 ([14]) Suppose that $(A, B)$ is controllable and $B \in \mathbb{R}^{n \times 1}$. Let the characteristic polynomial of $A$ be

$$
s^{n}+a_{n} s^{n-1}+\cdots+a_{1} .
$$

Define

Then there exists a $T$ such that

$$
\tilde{A}:=\left[\begin{array}{ccccc}
0 & 1 & & & \\
0 & 0 & & & \\
& & \ddots & & \\
& & & 0 & 1 \\
-a_{1}-a_{2} & & -a_{n-1}-a_{n}
\end{array}\right], \quad \tilde{B}:=\left[\begin{array}{c}
0 \\
\vdots \\
0 \\
1
\end{array}\right] .
$$

$$
T^{-1} A T=\tilde{A}, \quad T^{-1} B=\tilde{B} .
$$

The matrix pair $(\tilde{A}, \tilde{B})$ in Theorem 2 is said to be in controllable canonical form. Our procedure is the following.

(1) Convert the matrices (11), (15) into controllable canonical form using a nonsingular matrix $T_{1}$ from Theorem 2 .

(2) Pick a matrix $\tilde{J}$ so that $\tilde{J}_{\text {sym }}=\frac{1}{2}\left(\tilde{J}+\tilde{J}^{\top}\right)$ has all negative eigenvalues.

(3) Convert the matrices $\tilde{J}$ from step (2) and (15) into controllable canonical form using a non-singular matrix $T_{2}$ from Theorem 2 .

(4) Select the circuit parameters in the matrices from step (1) so that they equal the matrices from step (3).

Remark 1 Step (1) in the procedure above can be skipped if the state model for the PLL is derived directly from the transfer function (13) of the linearized system. In this case it possible to directly obtain a state model that is in controllable canonical form, see Example 4. 


\subsection{PLL with First Order Filter}

We now specialize our discussion to the case when $F(s)$ is a first order filter as in Examples 1 and 2 and take $N=1$ in the divide by $N$ counter. In this case (6) has state dimension $k=2, \sigma(X) \in \mathbb{R}^{2 \times 2}$ and $\sigma_{o}$ in (7) is a real-valued function.

Example 3 For the passive lead-lag filter of Examples 1 and 2 the computation (11) is given by

$$
J(x)=\left[\begin{array}{cc}
-\frac{1}{\tau_{1}+\tau_{2}} & 0 \\
\frac{\tau_{1}}{\left(\tau_{1}+\tau_{2}\right)^{2}} & 0
\end{array}\right]-K_{d}\left[\begin{array}{c}
1 \\
\frac{\tau_{2}}{\tau_{1}+\tau_{2}}
\end{array}\right]\left[\begin{array}{ll}
0 & K_{o}
\end{array}\right]=\left[\begin{array}{cc}
-\frac{1}{\tau_{1}+\tau_{2}} & -K_{o} K_{d} \\
\frac{\tau_{1}}{\left(\tau_{1}+\tau_{2}\right)^{2}} & -K_{o} K_{d} \frac{\tau_{2}}{\tau_{1}+\tau_{2}}
\end{array}\right] .
$$

Example 4 For the passive lead-lag filter the linearized system (12) is given by

$$
\begin{aligned}
\dot{\delta x}(t) & =\left[\begin{array}{cc}
-\frac{1}{\tau_{1}+\tau_{2}} & -K_{o} K_{d} \\
\frac{\tau_{1}}{\left(\tau_{1}+\tau_{2}\right)^{2}} & -K_{o} K_{d} \frac{\tau_{2}}{\tau_{1}+\tau_{2}}
\end{array}\right] \delta x(t)+\left[\begin{array}{c}
K_{d} \\
K_{d} \frac{\tau_{2}}{\tau_{1}+\tau_{2}}
\end{array}\right] \delta \theta_{1}(t) \\
\delta \theta_{e}(t) & =-\left[\begin{array}{ll}
0 K_{o}
\end{array}\right] \delta x(t)+\delta \theta_{1}(t) .
\end{aligned}
$$

The associated transfer function (13) is

$$
\frac{\Theta_{e}(s)}{\Theta_{1}(s)}=\frac{-\frac{K_{d} K_{o} \tau_{2}}{\tau_{1}+\tau_{2}} s-\frac{K_{d} K_{o}}{\tau_{1}+\tau_{2}}}{s^{2}+\frac{K_{d} K_{o} \tau_{2}+1}{\tau_{1}+\tau_{2}} s+\frac{K_{d} K_{o}}{\tau_{1}+\tau_{2}}}+1
$$

which has natural frequency

$$
\omega_{n}=\sqrt{\frac{K_{d} K_{o}}{\tau_{1}+\tau_{2}}}
$$

and damping ratio

$$
\zeta=\frac{1+K_{d} K_{o} \tau_{2}}{2 \sqrt{K_{d} K_{o}\left(\tau_{1}+\tau_{2}\right)}}
$$

To illustrate the ideas in Remark 1 , we find an alternate state model for the PLL starting from the above transfer function. The model is

$$
\begin{aligned}
& \dot{\delta} x(t)=\left[\begin{array}{cc}
0 & 1 \\
-\frac{K_{d} K_{o}}{\tau_{1}+\tau_{2}} & -\frac{K_{d} K_{o} \tau_{2}+1}{\tau_{1}+\tau_{2}}
\end{array}\right] \delta x(t)+\left[\begin{array}{l}
0 \\
1
\end{array}\right] \delta \theta_{1}(t)
\end{aligned}
$$

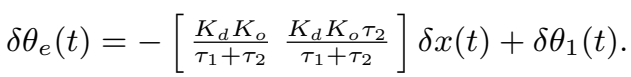

It is straight forward to show that both state models have the same input-output properties, i.e., the same transfer function.

To apply (14) to a PLL with a first order filter, we must apply two step inputs, $x$ and $y$. We treat $x$ as a step voltage signal, representing a phase or frequency step. Signal $y$ represents a zero phase or frequency step. However, as is illustrated in Figure 3, signal $y$ is a non-zero voltage. As a result, we see that both diffusion coefficients, $\sigma_{o}(x)$ and $\sigma_{o}(y)$, are non-zero and also one-dimensional. Thus, we can rewrite $\mathrm{SM}$ as

$$
\mathrm{SM}=\lambda_{0}|x-y|-\left|\sigma_{o}(x)-\sigma_{o}(y)\right|
$$




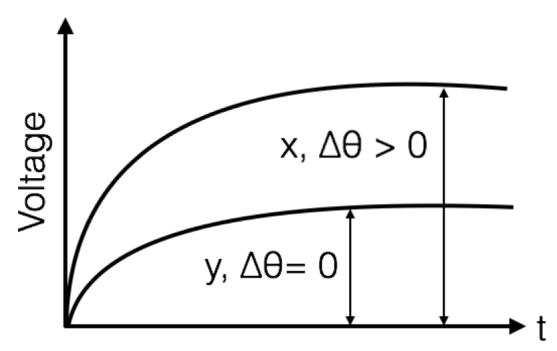

Fig. 3: Step Input Voltages.

We treat $\sigma_{o}^{2}$ as the variance parameter of the Brownian motion and relate it to some noise voltage, $n$, using the approximation

$$
\sigma_{o}^{2} t=n^{2}
$$

For simplicity, we now denote $\sigma_{o}(x)$ by $\sigma_{x}$ and $\sigma_{o}(y)$ by $\sigma_{y}$. The time parameter, $t$, in (17) is defined by the noise bandwidth. Thus, we can rewrite SM as

$$
\mathrm{SM}=\lambda_{0}|x-y|-\left|n_{x} \sqrt{f_{n x}}-n_{y} \sqrt{f_{n y}}\right| .
$$

In summary, to predict PLL stability using (18), we require two step voltages, $x$ and $y$, their respective noise voltages, $n_{x}$ and $n_{y}$, and their respective noise bandwidths, $f_{n x}$ and $f_{n y}$.

\section{Simulation Results}

\subsection{RLC Circuit}

We begin by demonstrating our approach on a simple series RLC circuit. This is an LTI system with state matrices

$$
A=\left[\begin{array}{cc}
0 & 1 / C \\
-1 / L & -R / L
\end{array}\right], \quad B=\left[\begin{array}{c}
0 \\
1 / L
\end{array}\right]
$$

Since the system is already linear, we have $J(x)=A$. The eigenvalues of $J_{\text {sym }}=$ $\frac{1}{2}\left(J+J^{\top}\right)$ are not all-negative even though $A$ is Hurwitz. Thus, we must make use of the procedure from Section 3.1.

We simulate this RLC circuit with a step input voltage, $V_{\text {in }}$, and solve it numerically as an SDE. ${ }^{3}$ Thus, we can directly define the diffusion term, $\sigma$, in (8) and use the definition of SM from (16).

Table 1 defines the circuit components and corresponding Lipschitz constraint, $\lambda_{0}$ obtained from (10). In Table 2 , we have listed the stability predictions using (14) for a constant diffusion term of $15 \mathrm{~V}$. In calculating SM, we need two inputs, $x$ and $y$, with corresponding diffusion terms, $\sigma_{x}$ and $\sigma_{y}$, respectively. In our calculations for the RLC circuit, we set $x=V_{\text {in }}$ and $y=0$. As a result, $\sigma_{x}$ is non-zero, while we treat $\sigma_{y}=0$. We will be measuring the voltage across the capacitor, $v_{C}(t)$. 
Table 1: Simulated RLC circuit configuration.

\begin{tabular}{cccc}
\hline $\mathrm{R}(\Omega)$ & $\mathrm{L}(\mathrm{mH})$ & $\mathrm{C}(\mathrm{nF})$ & $\lambda_{0}$ \\
\hline 100 & 40.91 & 97.68 & 7.5 \\
\hline
\end{tabular}

Table 2: RLC circuit stability predictions for a constant diffusion of $15 \mathrm{~V}$.

\begin{tabular}{cccc}
\hline$V_{\text {in }}(\mathrm{V})$ & $\sigma_{x}(\mathrm{~V})$ & $\mathrm{SM}$ & Stabiliy Prediction \\
\hline 0.1 & 15 & -14.3 & Unstable \\
2 & 15 & 0 & Marginally Stable \\
4 & 15 & 14.9 & Stable \\
\hline
\end{tabular}

Figure 4 shows the RLC circuit simulated at three different operating conditions. When $\mathrm{SM}<0$ in Figure $4 \mathrm{~b}$, the voltage across the capacitor, $v_{C}(t)$, shows significant oscillation, even when the noiseless simulation has already settled. When $\mathrm{SM}=0$ in Figure $4 \mathrm{~d}$, there is still some oscillation in $v_{C}(t)$; however, it appears to dwindle as the simulation continues. This is the marginally stable case, which acts, in some sense, as the threshold between stability and instability. Finally, when $\mathrm{SM}>0$ in Figure $4 \mathrm{f}, v_{C}(t)$ shows some ripple, but approaches steady-state near the end of the simulation time.

\subsection{Phase-Locked Loop}

We now present simulation results for four PLLs, each with the passive lead-lag loop filter shown in Figure 5 designed to have high loop gain and a multiplier phase detector. The PLL system parameters and component values are summarized in Tables $3 \mathrm{a}$ and $3 \mathrm{~b}$, respectively. The noise is lumped at the input of the phase detector.

The PLL configurations were determined using the methodology described in Section 3. Consequently, each of the configurations listed in Table 3 has a corresponding eigenvalue, $\lambda_{0}$ of $J_{\mathrm{sym}}$, that can be used in (14) to approximate the system's stability in the presence of noise.

Example 5 We demonstrate this stability margin calculation for PLL configuration 4. Since we know the passive lead-lag loop filter component values, we can calculate the time constants

$$
\tau_{1}=927.3 \mathrm{~ms}, \quad \tau_{2}=97.37 \mu \mathrm{s}
$$

Using the transfer function state model from Example 4, we write the relevant state matrices for PLL configuration 4

$$
\begin{gathered}
A=\left[\begin{array}{cc}
0 & 1 \\
-\frac{K_{d} K_{o}}{\tau_{1}+\tau_{2}} & -\frac{K_{d} K_{o} \tau_{2}+1}{\tau_{1}+\tau_{2}}
\end{array}\right]=\left[\begin{array}{cc}
0 & 1 \\
-400800340 & -40000
\end{array}\right] \\
B=\left[\begin{array}{l}
0 \\
1
\end{array}\right] .
\end{gathered}
$$

\footnotetext{
3 The circuit was simulated in MATALB ${ }^{\circledR}$ using the SDEtools library [15].
} 

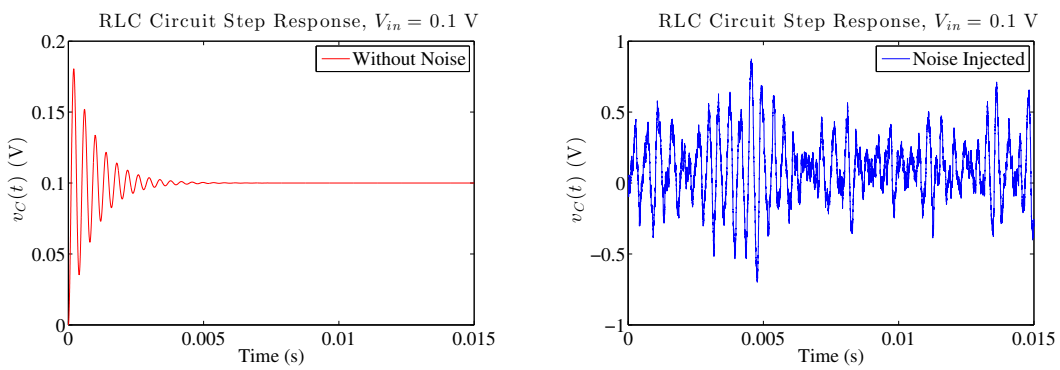

(a) Step input of $V_{\text {in }}=0.1 \mathrm{~V}$. Simulated without noise.

(b) Step input of $V_{\text {in }}=0.1 \mathrm{~V}$. Simulated with noise and unstable, $\mathrm{SM}=-14.3$.
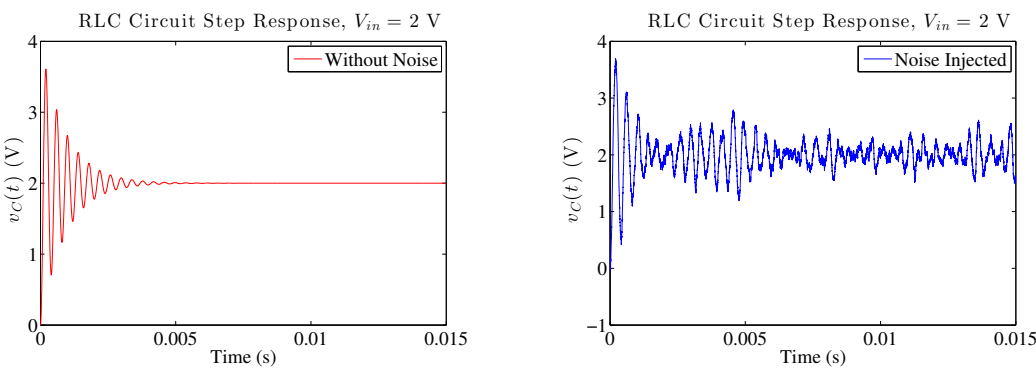

(c) Step input of $V_{\text {in }}=2 \mathrm{~V}$. Simulated without noise.

(d) Step input of $V_{\text {in }}=2 \mathrm{~V}$. Simulated with noise and marginally stable, $\mathrm{SM}=0$.
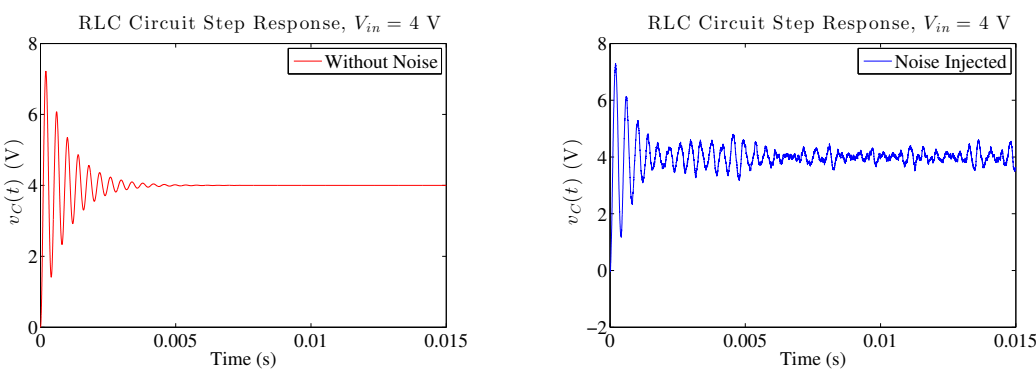

(e) Step input of $V_{\text {in }}=4 \mathrm{~V}$. Simulated without noise.

(f) Step input of $V_{\text {in }}=4 \mathrm{~V}$. Simulated with noise and stable, $\mathrm{SM}=14.9$

Fig. 4: RLC circuit simulations with $\sigma_{x}=15 \mathrm{~V}$.

We now assign $J=A$. Since $J_{\text {sym }}=\frac{1}{2}\left(J+J^{\top}\right)$ does not have all negative eigenvalues, we choose an arbitrary $\tilde{J}_{\text {sym }}$ that does, allowing us to define

$$
\tilde{J}=\left[\begin{array}{cc}
-20000 & 40017 \\
-20 & -20000
\end{array}\right], \quad \tilde{J}_{\mathrm{sym}}=\left[\begin{array}{cc}
-20000 & 19998.5 \\
19998.5 & -20000
\end{array}\right] \text {. }
$$




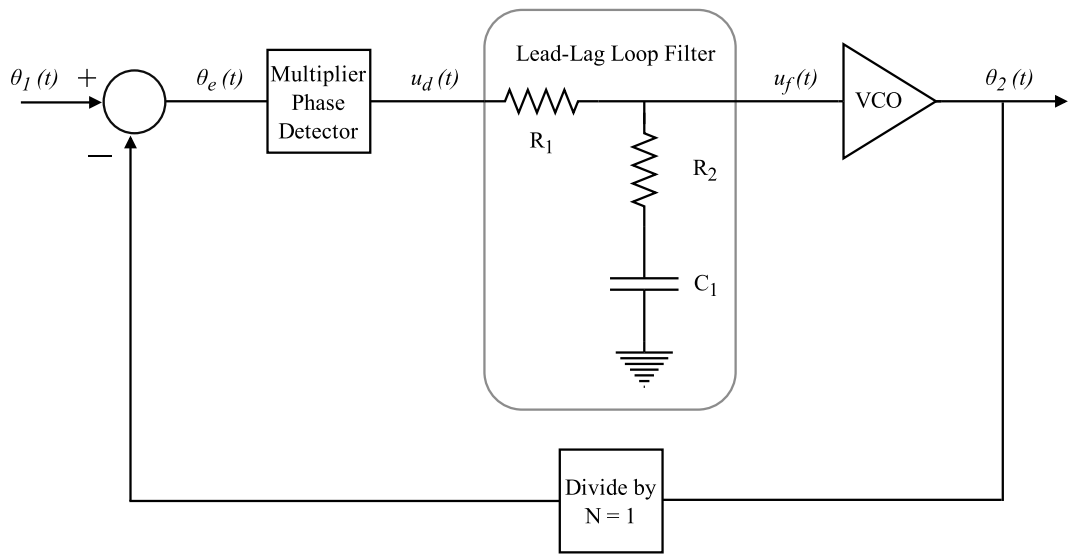

Fig. 5: Phase-locked loop with lead-lag loop filter and multiplier phase detector.

Table 3: Simulated PLL Configurations

(a) System Parameters

\begin{tabular}{cccc}
\hline Configuration & $\omega_{n}\left(\mathrm{rad} \cdot \mathrm{s}^{-1}\right)$ & $\zeta$ & $f_{3 \mathrm{~dB}}(\mathrm{~Hz})$ \\
\hline 1 & 2509.42 & 0.38 & 820.351 \\
2 & 2870.26 & 0.581 & 826.038 \\
3 & 2509.42 & 0.753 & 810.961 \\
4 & 20020 & 0.999 & 7655.97 \\
\hline
\end{tabular}

(b) Passive Lead-Lag Filter Component Values

\begin{tabular}{ccccc}
\hline Configuration & $K_{0} K_{d}\left(\mathrm{~s}^{-1}\right)$ & $R_{1}(k \Omega)$ & $R_{2}(k \Omega)$ & $C_{1}(\mathrm{nF})$ \\
\hline 1 & 24549 & 220 & 20 & 10 \\
2 & 34001.45 & 390 & 39 & 10 \\
3 & 39828.54 & 560 & 56 & 10 \\
4 & 410686 & 91 & 10 & 10 \\
\hline
\end{tabular}

$J$ and $\tilde{J}$ are related through $T^{-1} J T=\tilde{J}$, where

$$
T=\left[\begin{array}{lll}
40017 & 0 \\
20000 & 1
\end{array}\right] \text {. }
$$

Taking the eigenvalues of $\tilde{J}_{\text {sym }}$, we determine $-\lambda_{2}=-39998.5$ and $-\lambda_{1}=-1.5$, in order of size. Using (10) and the largest eigenvalue of $\tilde{J}_{\mathrm{sym}},-\lambda_{1}$, we calculate

$$
\lambda_{0}=\sqrt{2 \lambda_{1}}=1.5
$$

This value of $\lambda_{0}$ allows us to calculate SM for PLL configuration 4. To proceed, we must next choose the signal and noise values to be used in the simulation.

In Table 4, we have chosen a constant noise voltage, $n_{x}$, of $33 \mathrm{mV} / \sqrt{\mathrm{Hz}}$. We have chosen to restrict the noise bandwidth to half of the PLL's loop filter bandwidth, $f_{3 \mathrm{~dB}}$. Since each PLL configuration has a different bandwidth, $\sigma_{x}$ is not 
Table 4: PLL stability predictions for constant noise voltage of $33 \mathrm{mV} / \sqrt{\mathrm{Hz}}$.

\begin{tabular}{ccccc}
\hline Configuration & System Poles & $\lambda_{0}$ & $\sigma_{x}(\mathrm{~V})$ & Stability \\
\hline 1 & $-1222.22 \pm \jmath 2956.89$ & 7.5 & 0.668 & Above 37.7 dB \\
2 & $-1666.67 \pm \jmath 2336.79$ & 12.55 & 0.671 & Above 37.6 dB \\
3 & $-1888.89 \pm \jmath 1652.06$ & 4.44 & 0.665 & Above 37.7 dB \\
4 & $-20000 \pm \jmath 894.62$ & 1.73 & 2.042 & Above 38.5 dB \\
\hline
\end{tabular}

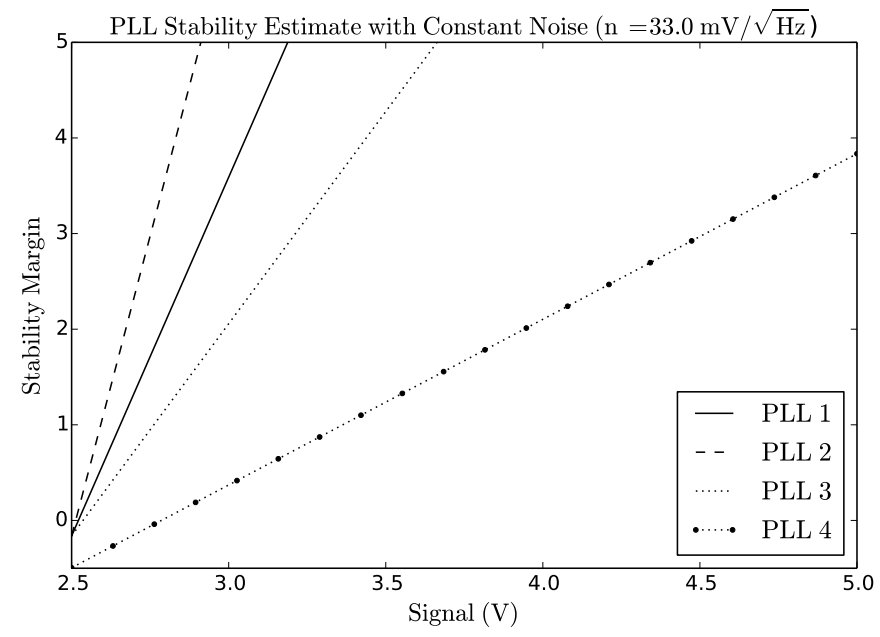

Fig. 6: Predicted stability for a constant noise voltage of $33 \mathrm{mV} / \sqrt{\mathrm{Hz}}$ for all four PLL configurations. Here we have plotted the desired stability margin, SM, against the allowable input signal, $x$, from (18). Although the noise voltage is held constant, as we change the input signal, the noise bandwidth, $f_{n x}$, also changes. Thus, by sweeping SM to determine the corresponding $x$, we are also varying the quantity of noise, $\sigma_{x}$.

constant across configurations. We have listed the PLL poles and eigenvalue, $\lambda_{0}$, to illustrate the PLL's proximity to the right half-plane relative to the measure of noise. Finally, we have listed the minimum SNR at which (14) predicts the PLL to be stable $(\mathrm{SM}>0)$. Since the noise voltage, and thus $\sigma_{x}$, is held constant in Table 4 , we are varying the step voltage input, $x$. Additionally, since signal $y=2.5$ $\mathrm{V}$ for a zero frequency or phase step, we can calculate $n_{y}$ to be $25 \mathrm{mV} / \sqrt{\mathrm{Hz}}$.

Table 4 predicts that as the PLL's poles move farther into the left half-plane, the PLL can generally tolerate a higher quantity of noise, represented by $\sigma$. This is an expected result, since this represents increased robustness of the PLL's stability. This trend is most obvious between PLL configurations 3 and 4 ..

We have simulated these PLL configurations using the PLL Design and Simulation software provided in [1]. This software allows us to input a frequency or phase step, choose an SNR and define a relative noise bandwidth. We have plotted the four PLLs' expected stability from (14) in Figure 6, where the minimum input step voltage ( $y$ in Figure 3 ) is $2.5 \mathrm{~V}$, analogous to a step input of $0 \mathrm{~Hz}$. There 


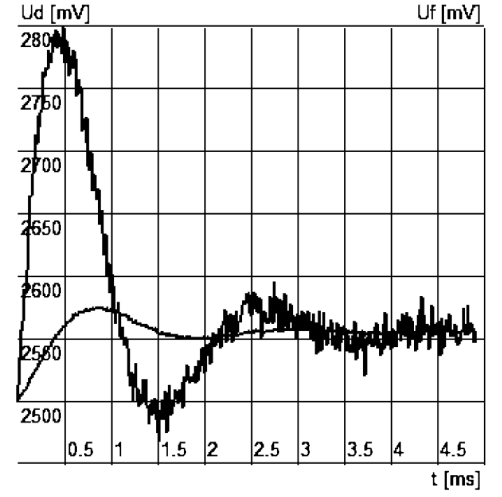

(a) PLL configuration 1 simulated with noise and $f_{\text {step }}=170 \mathrm{~Hz}$. The $\mathrm{PLL}$ is stable with $\mathrm{SM}=2$.

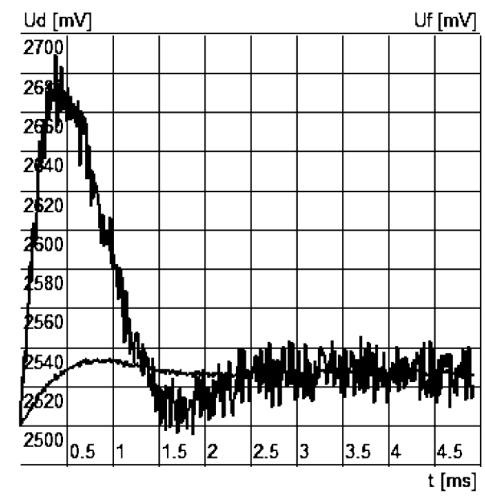

(c) PLL configuration 2 simulated with noise and $f_{\text {step }}=115 \mathrm{~Hz}$. Stable with $\mathrm{SM}=2$.

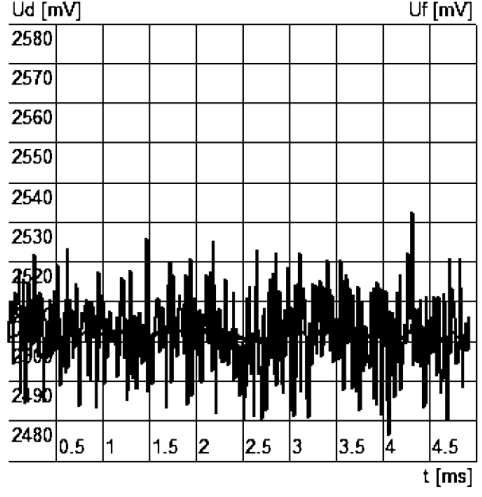

(b) PLL configuration 1 simulated with noise and $f_{\text {step }}=5 \mathrm{~Hz}$. Unstable with $\mathrm{SM}=-0.11$.

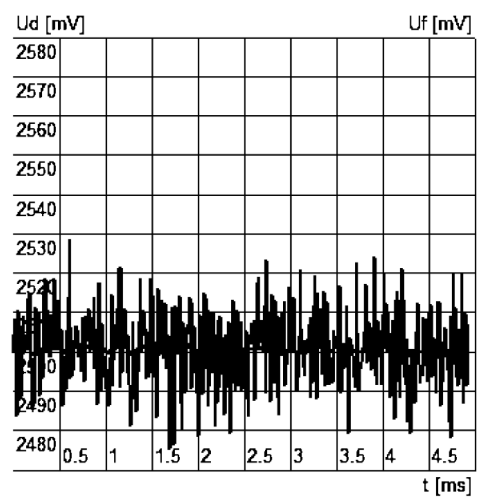

(d) PLL configuration 2 simulated with noise and $f_{\text {step }}=4 \mathrm{~Hz}$. Unstable with $\mathrm{SM}=-0.13$.

Fig. 7: PLL simulations at $\mathrm{n}=33 \mathrm{mV} / \sqrt{\mathrm{Hz}}$.

is significant variation in the stability estimate for the four configurations. As a result, the SM simulation conditions chosen in Figure 6 vary between PLL configurations, specifically when $\mathrm{SM}<0$ to ensure that $x \approx y$. Additionally, simulation conditions for PLL configuration 4 were selected based on the maximum frequency step that did not introduce additional distortion.

Table 5 describes the simulation conditions for PLL configuration 4. Given SM $<0$, we expect the PLL to oscillate, as illustrated in Figure $7 \mathrm{~h}$. To verify that the signal distortion present on the phase detector output voltage, $U_{d}$, is a result of oscillation and not superimposed noise, we have calculated the apparent SNR. Since this value exceeds the input SNR by $3.93 \mathrm{~dB}$, we can attribute the distortion to instability. At $\mathrm{SM}=1.5$ in Figure $7 \mathrm{~g}$, the PLL is obviously stable. Given the fact that the system poles in Table 3 for PLL configuration 4 are so deep into the left half-plane, we expect this configuration to be the most stable. 


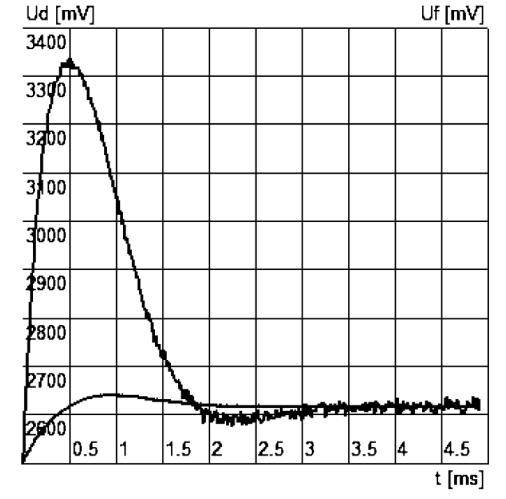

(e) PLL configuration 3 simulated with noise and $f_{\text {step }}=580 \mathrm{~Hz}$. Stable with $\mathrm{SM}=2$.

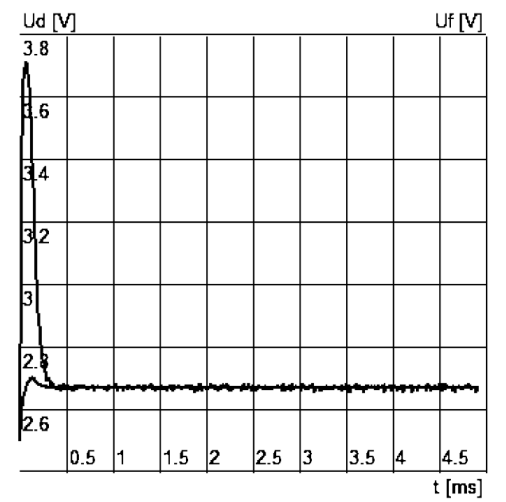

(g) PLL configuration 4 simulated with noise and $f_{\text {step }}=8700 \mathrm{~Hz}$. Stable with $\mathrm{SM}=1.5$.

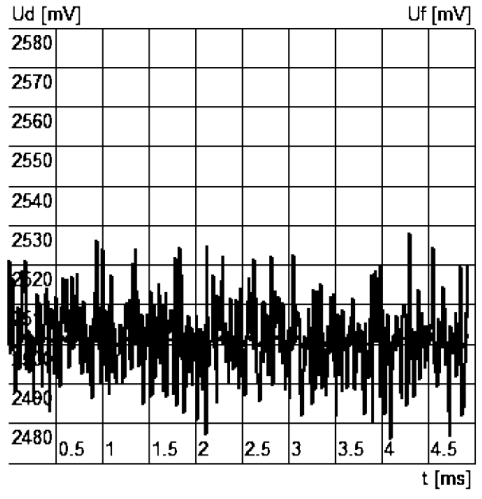

(f) PLL configuration 3 simulated with noise and $f_{\text {step }}=4 \mathrm{~Hz}$. Unstable $\mathrm{SM}=-0.13$.

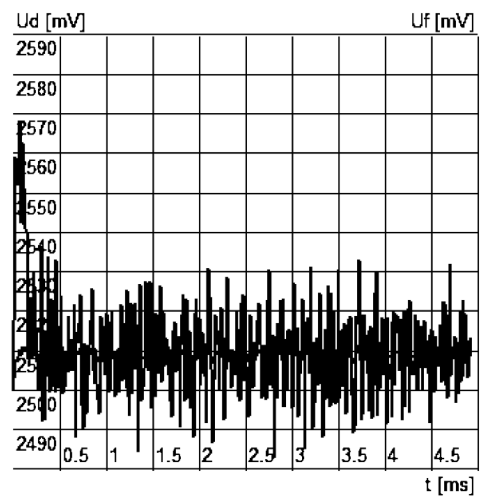

(h) PLL configuration 4 simulated with noise and $f_{\text {step }}=460 \mathrm{~Hz}$. Unstable with $\mathrm{SM}=-0.4$.

Fig. 7: PLL simulations at $\mathrm{n}=33 \mathrm{mV} / \sqrt{\mathrm{Hz}}$ (continued).

Figures $7 \mathrm{~b}, 7 \mathrm{~d}$, and $7 \mathrm{f}$ show PLL configurations 1, 2 and 3, respectively, with $\mathrm{SM}<0$. The step input in these simulations is very small and $x \approx y$ from Figure 3. Consequently, we expect the PLL to settle very quickly under these conditions. Instead, we see significant ripple on the signal $U_{d}$. As illustrated for PLL configuration 4 in Table 5, this ripple is oscillation caused by the injected noise.

The simulations with $\mathrm{SM}>0$ show $U_{d}$ settling, albeit with extra noise caused by the reduced SNR. The disparity between simulations with $\mathrm{SM}<0$ and $\mathrm{SM}>$ 0 illustrates the effectiveness of (14), even with approximations like (17) and the limitations imposed by the simulation software. 
Table 5: PLL configuration 4 simulation results for constant noise voltage of 33 $\mathrm{mV} / \sqrt{\mathrm{Hz}}$

\begin{tabular}{ccc}
\hline Parameter & $\mathrm{SM}=-0.4$ & $\mathrm{SM}=1.5$ \\
\hline Stability Estimate & Unstable & Stable \\
Signal Step & $2.56 \mathrm{~V}$ & $3.65 \mathrm{~V}$ \\
Frequency Step & $460 \mathrm{~Hz}$ & $8700 \mathrm{~Hz}$ \\
Input SNR & $37.79 \mathrm{~dB}$ & $41.55 \mathrm{~dB}$ \\
Apparent SNR & $41.72 \mathrm{~dB}$ & - \\
\hline
\end{tabular}

\section{Conclusions}

This paper presents a new figure of merit, the stability margin, that is used to predict PLL stability in the presence of noise. This quantity is calculated based on the asymptotics of singular diffusions for a PLL with a multiplier type phase detector and a passive lead-lag filter. Given a voltage input step and the quantity of input-referred noise, the stability margin can be analytically calculated for a PLL. Simulation results for a series RLC circuit and four PLL configurations demonstrate the stability margin predicting the onset of instability. The methodology presented in this paper is expected to assist in PLL design by providing an accessible assessment of stability in the presence of noise.

Acknowledgements We thank Professor Andrew Heunis for bringing Theorem 1 to our attention.

\section{References}

1. R. E. Best, Phase-locked loops: Theory, design and applications. McGraw Hill, 6th ed., 1993.

2. Z. Shu, K. Lee, and B. H. Leung, "A 2.4-GHz ring-oscillator-based CMOS frequency synthesizer with a fractional divider dual-PLL architecture," IEEE Journal of Solid-State Circuits, vol. 39, no. 3, 2004.

3. S. Ryu, H. Yeo, Y. Lee, S. Son, and J. Kim, "A $9.2 \mathrm{GHz}$ digital phase-locked loop with peaking-free transfer function," IEEE Journal of Solid-State Circuits, vol. 49, no. 8, 2014.

4. A. Sai, Y. Kobayashi, S. Saigusa, O. Watanabe, and T. Itakura, "A digitally stabilized type-III PLL using ring VCO with 1.01psrms integrated jitter in 65nm CMOS," in 2012 IEEE International Solid-State Circuits Conference Digest of Technical Papers (ISSCC), 2012.

5. W. E. Ali, C. Hedayat, and C. Hangmann, "Simulative characterization of the stability for second order voltage switched CP-PLL," in 2013 IEEE 56th International Midwest Symposium on Circuits and Systems (MWSCAS), pp. 153-156, 2013.

6. Y. Song, H. Fu, H. Yu, and G. Shi, "Stable backward reachability correction for PLL verification with consideration of environmental noise induced jitter," in 2013 18th Asia and South Pacific Design Automation Conference (ASP-DAC), pp. 755-760, January 2013.

7. A. Mehrotra, "Noise analysis of phase-locked loops," IEEE Transactions on Circuits and Systems -I: Fundamental Theory and Applications, vol. 49, no. 9, pp. 1309-1316, 2002.

8. B. G. Gawalwad and S. N. Sharma, "On a perturbed phase-locked loop system: A simple physical model," in 2013 IEEE International Conference on Control Applications (CCA), pp. 430-436, IEEE, August 2013.

9. R. N. Bhattacharya and E. C. Waymire, Stochastic Processes with Applications, vol. 61. Philadelphia, PA, USA: SIAM, 1990 
10. D. Abramovitch, "Lyapunov resdesign of analog phase-lock loops," IEEE Transactions on Communications, vol. 38, no. 12, pp. 2197-2202, 1990.

11. A. Hajimiri, "Noise in phase-locked loops," in 2001 Southwest Symposium on Mixed-Signal Design, 2001. SSMSD., 2001.

12. B. Oksendal, Stochastic Differential Equations: An Introduction with Applications. Springer-Verlag, 1998.

13. R. Z. Has'minskii, Stochastic stability of differential equations. Sijthoff \& Noordhoff, 1980

14. W. Wonham, Linear Multivariable Control: A Geometric Approach. New York: SpringerVerlag, 3rd ed., 1985.

15. A. Horchler, "SDETools," November 2013. 